\title{
Evaluation of the Permeation and Penetration of Two Formulations of Terbinafine Chlorhydrate Incorporated in Liposomes (Cream 1\%) vs. a Conventional Formulation (Cream 1\%), in an in Vitro-ex Vivo Model
}

\author{
Sanabria Kinski ${ }^{1}$, Annunziato Maria Antonieta2 ${ }^{2}$, Chacon Nicolas ${ }^{2}$, Inatti Alfredo ${ }^{2}$ \\ ${ }^{1}$ Department of Nanotechnology of Laboratories Leti S.A.V., Guarenas, Venezuela \\ ${ }^{2}$ Department of Research and Development, Laboratories Biocontrolled C.A., Guarenas, Venezuela \\ Email: servi.biocontrolled@gmail.com
}

How to cite this paper: Kinski, S., Antonieta, A.M., Nicolas, C. and Alfredo, I. (2019) Evaluation of the Permeation and Penetration of Two Formulations of Terbinafine Chlorhydrate Incorporated in Liposomes (Cream 1\%) vs. a Conventional Formulation (Cream 1\%), in an in Vitro-ex Vivo Model. Journal of Biosciences and Medicines, 7, 119-133.

https://doi.org/10.4236/jbm.2019.78010

Received: May 30, 2019

Accepted: August 27, 2019

Published: August 30, 2019

Copyright ( 2019 by author(s) and Scientific Research Publishing Inc. This work is licensed under the Creative Commons Attribution International License (CC BY 4.0).

http://creativecommons.org/licenses/by/4.0/

\begin{abstract}
In this trial, a topical formulation of a $1 \%$ liposome $\mathrm{TH}$ cream was compared to a conventional $\mathrm{TH}$ formulation. Permeation was evaluated through a synthetic membrane. The study provided information on the rate of diffusion and percentage of drug release and also compared the penetration dynamics in the layers of the stratum corneum of both formulations. Liposome TH cream was able to deliver the active ingredient to a larger number of SC layers, with a higher amount of drug, which is very promising for the treatment of superficial mycoses.
\end{abstract}

\section{Keywords}

Antifungal, Franz Diffusion Cell, Tape Striping Method, Liposome, Terbinafine $\mathrm{HCl}$, Drug Carrier Systems, Stratum Corneum

\section{Introduction}

Superficial mycoses are infections caused by different groups of fungi that are pathogenic to humans, which invade keratinized structures such as stratum corneum (SC), hair, nails, and/or mucous membranes. Topical therapy is an attractive option for the treatment of skin infections due to certain advantages such as the targeting of drugs at the site of infection and the reduction of the risk of systemic side effects [1] [2]. 
$\mathrm{TH}$ is an antifungal agent of the allylamine class that selectively inhibits the enzyme squalene-2-3-epoxidase, interfering in the early stage of the synthesis of ergosterol, a fundamental component of the fungal membrane, leading to a deficiency of ergosterol and an accumulation of squalene, resulting in cell death. TH in topical cream is indicated in the treatment of superficial fungal infections of the skin, scalp, and nails such as: Tinea corporis, Tinea cruris, Tinea pedis, Tinea capitis, and Onychomycosis. SC is the main site of action of $\mathrm{TH}$ in superficial fungal skin infections [1] [3] [4]. The minimum inhibitory concentration (MIC) of $\mathrm{TH}$ is $0.001-0.01 \mu \mathrm{g} / \mathrm{mL}$ and the minimum fungicidal concentration is 0.003 $0.006 \mu \mathrm{g} / \mathrm{mL}$. The drug must be present at a concentration above its MIC in the target site for a guaranteed therapeutic effect [5]. SC is a permeability barrier with a high lipophilic potential that hinders the penetration of drugs into skin and is structured as a network where the corneocytes, which are rich in keratin, are located at the intercellular matrix that is provided with lipids. From this structure, dermal permeation of compounds takes place through the transcellular, intercellular and/or through cutaneous annexes. The effectiveness of the drug will depend on its penetration and concentration at the level of the target layers of the skin [6] [7].

The administration of antifungals to the target site of the skin is a great challenge in therapeutic terms, since antifungal drugs are generally highly lipophilic compounds, which hinders the penetration of the drug through the SC. The solubility of TH in water is very poor and is also very lipophilic [6] [7] [8]. The use of topical nanoscale Drug Carrier Systems (DCS) may be a potential option to increase the penetration and concentration of the drug in the different layers of the skin, as they can pass through pores and cell membranes. The nanoscale DCS most used is liposomes due to their structural components (cholesterol and/or phospholipids) which allow it to be biocompatible, biodegradable and easily metabolized by the body. On the other hand, their elasticity and size when applied topically allows them to overcome the skin barriers, managing to transport and deliver the active principle in the action site without affecting its composition, structure and pharmacokinetics. Under certain circumstances (incomplete or defective SC) the topical liposomes are able to come into direct contact with the keratinocytes of the deeper layers of the epidermis [6] [7] [9] [10].

\section{Objectives}

To evaluate the permeation-via Franz diffusion cell test-and the penetrationvia tape striping method-of two antifungal formulations of $\mathrm{TH}$ incorporated in liposomes (cream 1\%) vs a conventional no liposome formulation (Lamisil ${ }^{\circledR} 1 \%$ cream).

\section{Methods}

In this trial, a topical formulation of a $1 \%$ liposome $\mathrm{TH}$ cream (test) was compared to a conventional no liposome TH formulation (Lamisil ${ }^{\circledR} 1 \% \mathrm{cream}$ ), com- 
mercialized in Venezuela. Permeation was evaluated in terms of diffusion through a synthetic membrane (In Vitro test/Franz cells). Penetration and quantification were evaluated in skin (Ex vivo test/Tape Stripping method). The 1\% liposome cream sample was characterized physicochemically.

\subsection{Liposome Preparation}

The liposome cream was formulated by creating vesicles prepared by mixing the surfactant, phospholipids and the active principle. Then the dispersion of the vesicles was carried out in a $0.5 \%$ sodium chloride solution with the aid of a mechanical stirrer to homogenize the mixture. The vesicles formed spontaneously upon contact with the aqueous solution. Thus the buffer was trapped in the lipid bilayer and the rest immersed in the interior of the liposome. Initially, mixed micelles were formed.

\subsection{Characterization and Evaluation of the Formulation}

The physicochemical characterization of the liposome cream was carried out, evaluating the color, odor, appearance, $\mathrm{PH}$ value and density.

1) Determination of the particle size of the suspension (Test Formulation): The particle size of the cream was obtained using the Zetasizer Nano model ZS equipment from Malvern Instrument (USA). This equipment operates at a wavelength of $632 \mathrm{~nm}$ and a maximum power of $10 \mathrm{~mW}$. It determines the particle size by averaging the rate of fluctuations in the intensity of laser light scattered by the particles as they diffuse into a fluid. To measure the size and polydispersity index, $200 \mu \mathrm{L}$ of the cream was placed in $800 \mu \mathrm{L}$ of $0.1 \%$ sodium chloride solution. A glass cuvette was used and the measurements were recorded. Subsequently, the average of all determinations was made.

- An aliquot of liposome suspension and $0.1 \%$ sodium chloride solution was taken.

- It was mixed in a glass cell maintaining the solution at high temperature.

- The sample was read on the Nano Zetasizer.

- The sample reading conditions were solvent of the $0.1 \% \mathrm{NaCl}$ sample and the equipment temperature $70^{\circ} \mathrm{C}$.

This experiment was carried out in triplicate.

2) Determination of the Zeta potential of the liposome suspension (Test Formulation): It was determined using the Zetasizer Nano model ZS equipment from Malvern Instrument (USA), which operates at a wavelength of 632 $\mathrm{nm}$ and at a maximum power of $10 \mathrm{~mW}$. The equipment is able to estimate the electrostatic interaction between the particles, thus indicating the stability of the dispersion. This equipment uses the Laser Doppler Electrophoresis (LDE) technique that assumes the frequency variation of any type of wave emitted or received by a particle in motion. The sample used to determine particle size and polydispersity index was placed in a zeta cell and potential measurements were made.

- An aliquot of liposome suspension and $0.1 \%$ sodium chloride solution was 
taken.

- They were mixed and placed in a suitable cell to estimate zeta potential.

- The sample reading conditions were solvent from the $0.1 \% \mathrm{NaCl}$ sample, equipment temperature $70^{\circ} \mathrm{C}$.

Subsequently, an average of all the determinations was made.

3) Determination of the $\mathrm{pH}$ value: The $\mathrm{pH}$ was determined at $25^{\circ} \mathrm{C}$ using a $\mathrm{pH}$ meter brand Gehaka. First, the electrode was calibrated with Standard Buffer solutions of $\mathrm{pH} 4.0$ and $\mathrm{pH}$ 6.0. The electrode was placed in the cream until stabilized. The determination was made in triplicate.

4) Determination of viscosity: The viscosity was determined using a Brookfield viscometer equipped with a suitable needle. The determination was made with the sample at room temperature and was carried out in triplicate.

5) Determination of Total TH: The determination of the total percentage of $\mathrm{TH}$ in the cream was made by dissolving $250 \mathrm{mg}$ of cream in methanol, then the solubilized drug was filtered through a $0.45 \mu \mathrm{m}$ membrane and its absorbance was determined by HPLC at a length of $280 \mathrm{~nm}$.

6) Determination of the percentage of encapsulated TH (Test Formulation): It was carried out using the $3 \mathrm{~mL}$ molecular exclusion columns constructed with Sephadex G-25. The determination was made with $250 \mu$ of sample that was passed through the column with several washes of a $0.1 \%$ sodium chloride solution.

a) Treatment of the $\mathrm{TH}$ standard $(0.05 \mathrm{mg} / \mathrm{mL})$ :

- The quantification standard prepared for the determination of the total content of TH described in step 2 was used.

b) Treatment of the sample for the determination of encapsulated active principle:

- An eluted volume was weighted in each vial collected by separation by molecular exclusion in a suitable balloon.

- Methanol was added and placed on ultrasound for 30 minutes.

- It was expected to reach room temperature and made up to the mark with methanol.

- The sample collected subsequently was dissolved in methanol and filtered through a $0.45 \mu \mathrm{m}$ membrane directly into the vial.

The vials in which a positive absorbance value was found in the phosphate sample are those that contribute to the quantification of the encapsulated active principle. Its absorbance was determined by HPLC at a length of $280 \mathrm{~nm}$.

\subsection{Determination of the Polydispersity Index of the Suspension (Test Formulation)}

- The same dilution and equipment conditions were used to estimate the particle size of the suspension.

\subsection{TH Permeability Studies in Vitro (Diffusion in Franz Cells)}

The permeation technique was used through a dialysis membrane used for in 
vitro permeation methods. The receptor medium was filled with a Saline Phosphate Buffer pH 5.8: Ethanol (1:2) previously sonicated and degassed. The dialysis membrane SIGMA brand of 12,500 Da of porosity, previously activated, was used. Subsequently, $0.035 \mathrm{~g}$ of cream was placed in the donor compartment. The system was maintained at suitable temperature and agitation. Aliquots were removed at intervals of $0,0.5,1,2,3,24$ hours and the volume was immediately replaced after each sampling. The content of the drug was determined by HPLC at a wavelength of $280 \mathrm{~nm}$.

Diffusion assays such as the Franz Cells are in vitro studies to evaluate transepithelial penetration and drug release over time. The Franz cells constitute a system composed of two chambers, a superior compartment (donor) where the analyte is placed and a lower compartment (receptor) with the diffusion medium, separated by a membrane of animal, human or synthetic origin that allows the evaluation of the diffusion of biologically active molecules from one chamber to another. The release of the active principle is controlled by a diffusion process based on a concentration gradient from the donor compartment to the receptor compartment. In the upper compartment, a solution or dispersion containing the active compound is added and in the lower one the corresponding samples are taken, which are subsequently quantified by analytical techniques such as UV and HPLC [11] [12] [13]. For this study, diffusion trials were carried out using Franz cells Hanson Research Model 58-001-430. In this essay, a sample of each one of the formulations of TH 1\% cream was placed in the Franz diffusion cell. A permeation profile was performed at times 0 minutes, 30 minutes, 1 hour, 2 hours, 4 hours and 24 hours. The measurements were made by high performance liquid chromatography (HPLC).

\section{1) Calculation of the speed and amount of Freed Drug.}

- The amount released $\left(\mu \mathrm{g} / \mathrm{cm}^{2}\right)$ was calculated at a given time for each sample.

- Quantity released at time zero.

For each cell, the individual amount of the drug released was plotted based on the square root of time.

\section{2) Calculation of $\%$ of Drug Released in Time:}

- The percentage of active principle released in each of the times evaluated in the diffusion profile was calculated for each sample.

- For each cell, the percentage of drug released as a function of time was plotted.

\subsection{TH ex Vivo Permeation Studies (Tape Stripping Method)}

This study was conducted with pig's ear skin. The penetration of the active principle in the different layers of the SC of the skin and the VE was evaluated. The pig's ear skin was previously washed, the bristle-like hair was removed and the fat was removed from the skin. The skin samples were washed with normal saline and cut into the appropriate sizes. The skin was placed on a Saarbrücker support and $300 \mathrm{mg}$ of cream was placed on it. Then the oven was kept at $37^{\circ} \mathrm{C}$ 
for 1 hour and then the excess cream was cleaned on the skin and each layer of the SC was removed with adhesive tapes. To remove each layer of the SC, an adhesive tape was placed for 10 seconds with a weight of $2 \mathrm{~kg}$. Each tape was placed in a $15 \mathrm{~mL}$ falcon tube and $5 \mathrm{~mL}$ of methanol was added. A total of 20 tapes were collected for each layer of the SC. Additionally, the VE was placed in a $15 \mathrm{~mL}$ falcon tube and $5 \mathrm{~mL}$ of methanol was added. All samples were analyzed by HPLC at a wavelength of $280 \mathrm{~nm}$. The mass of TH present in each layer of SC and VE was graphed.

The penetration was evaluated by means of an ex vivo test/Tape Stripping Method (TS), using Scotch 3M $18 \mathrm{~mm}$ magic adhesive tape strip (USA). The TS method evaluates the ex vivo or in vivo penetration of an active principle found in a topical pharmaceutical form at different skin layers and at different times. [14] [15]. The foundation of the TS consists essentially in theoretically removing a layer of corneocytes with an adhesive tape to the skin, that is to say, the test is carried out by means of the consecutive elimination of layers of subcutaneous tissue or hypodermis ( $0.5-1.0$ microns) with adhesive tapes on the surface of the skin. It has been found that around 20 strips of tape are needed to strip most of the SC [16]. Good correlation of in vitro results with in vivo results has been demonstrated for a wide variety of substances when using TS methods and Diffusion cells [17]. In this essay, an amount of $300 \mathrm{mg}$ of $1 \% \mathrm{TH}$ cream (of each formulation) was placed on a $25 \mathrm{~mm}$ diameter pig ear skin disk. The strips were removed after completing $60 \mathrm{~min}$ in incubation at a controlled temperature of $37^{\circ} \mathrm{C}$, and then extracted one by one placing a weight of $2 \mathrm{~kg}$ on the tape for 10 seconds. A total of 21 adhesive tapes were evaluated, of which the first 20 tapes correspond to samples from the SC and the number 21 corresponds to sampling of the viable epidermis (VE). The speed and amount of the drug that penetrated the SC were calculated through the equation of the line with the calibration curve made with the TH pattern. The mass of the active ingredient was quantified for each of the tapes and the skin analyzed by each disc and the mass of the active principle present in each layer of the SC was then plotted per disc analyzed. The measurements of the product were made by HPLC.

\subsection{Type of Sample Used in the ex Vivo Test}

For studies of this nature, pig skin represents an appropriate model ex vivo to predict the behavior of a topical administration system, because it has histological and structural similarities with human skin. In animal models it is estimated that pig skin is the one with permeability characteristics more similar to human skin (Meyer et al., 1978a [18], Schmook et al., 2001 [19]), besides being considered as a study model for topical absorption (Epicutaneous or transdermal) of drugs for Neonates (Sekkat y col., 2004) [20].

\subsection{Statistical Analysis}

The statistical analysis should answer the following questions and hypotheses to corroborate if the difference between both formulations Test vs. Reference are 
statistically significant in the ex vivo study. Taking into account the type of variables that were being compared the hypotheses to answer this question were the following:

- Ho: There are no differences between the amount of drug absorbed by the SC and skin between both pharmaceutical formulations.

- H1: There are differences between the amount of drug absorbed by the SC and skin between both pharmaceutical formulations.

We proceeded to make a comparison of the arithmetic means of the total amount absorbed by the SC and the skin of the pig of each product in each disc (6 per group) after supplying $300 \mathrm{mg}$ of the cream to $1 \%$ containing $3 \mathrm{mg}$ of $\mathrm{TH}$, and then one hour of incubation, by unpaired Student's T. To ensure the normality of the data, we proceeded to its logarithmic transformation (LT).

\section{Results}

The sample was a bright white, unctuous, and fast absorbing cream. With the help of the method of separation by molecular exclusion it was possible to determine the percentage of encapsulation of the $\mathrm{TH}$ in the emulsion, which was $92.8 \%$ thus ensuring that a large part of the active principle is encapsulated within the vesicles formed. Table 1 summarizes the physicochemical characterization of the product.

Table 1. Physicochemical characterization of liposome test formulation.

\begin{tabular}{cc}
\hline PARTICLE SIZE $(\mathrm{nm})$ & 72.25 \\
POLIDISPERSITY INDEX & 0.179 \\
ZETA POTENTIAL (mV) & 13.3 \\
DENSITY $(\mathrm{g} / \mathrm{mL})$ & 1.0117 \\
pH VALUE & 2.15 \\
\hline
\end{tabular}

\subsection{In Vitro Test (Diffusion in Franz Cells)}

Comparing the release profiles of the two formulations evaluated, a higher diffusion rate (Figure 1) and a greater percentage released of the active ingredient were observed in the liposome test formulation (Figure 2).

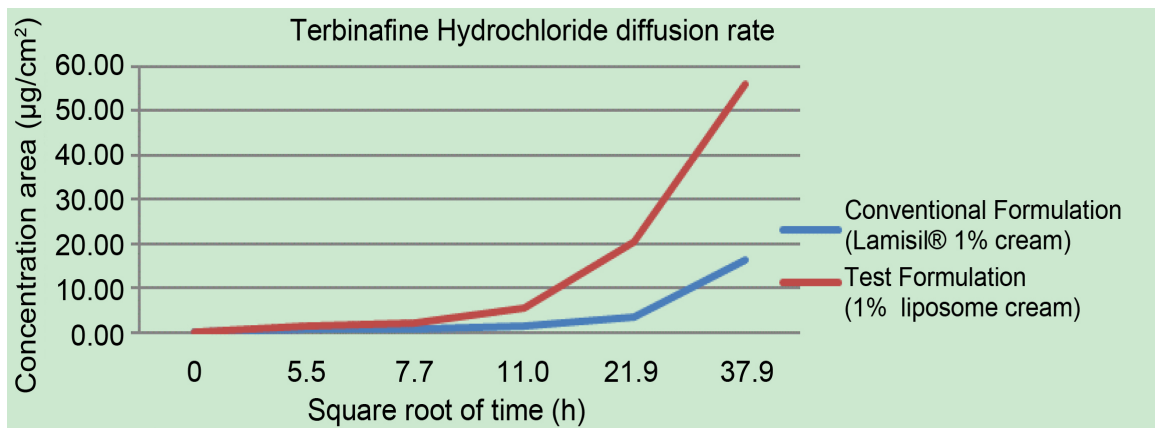

Figure 1. Terbinafine hydrochloride diffusion rate as a function of square root of time for the conventional no liposome formulation vs. liposome test formulation. 


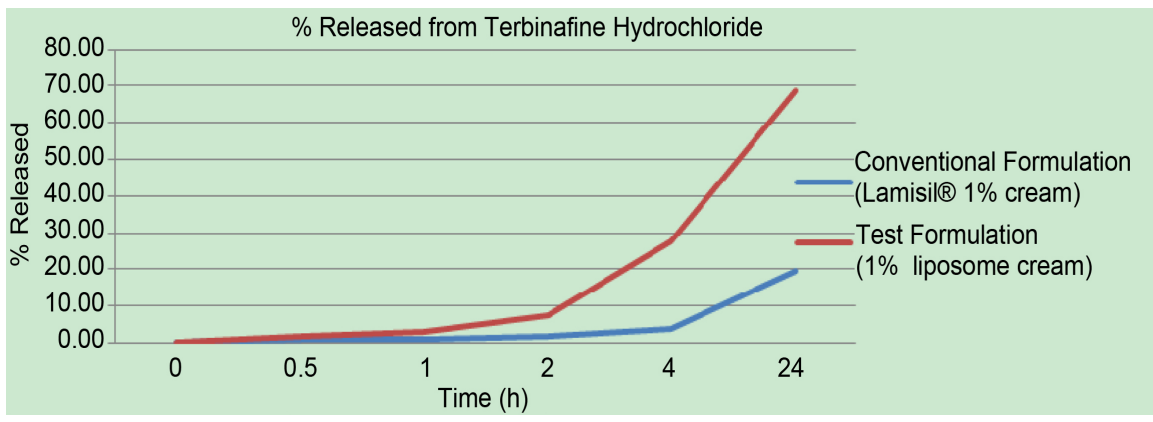

Figure 2. Percentage released from Terbinafine Hydrochloride as a function of time for the Conventional no liposome Formulation vs. liposome test formulation.

\subsection{Ex Vivo Test (Tape Stripping)}

Table 2 shows that both formulations are present in a large part of the adhesive tapes tested, being the liposome test formulation the one most frequently found in the SC and the VE. When the mean TH values were calculated for each adhesive tape, it was found that the drug quantities were highly adequate and above the range established for the MIC $(0.001-0.01 \mu \mathrm{g} / \mathrm{mL})$ to achieve in situ concentrations, finding that the average concentration of the Test formulation was superior to that of the Conventional formulation (104.75 $\mu \mathrm{g}$ vs. $50.32 \mu \mathrm{g}$ respectively). The liposome test formulation achieved a greater number of layers of the SC, and also exceeded in mass the Conventional formulation, reaching inclusively the VE.

Table 2. Total values of TH absorbed per disc after one hour of incubation $(\mu \mathrm{g})$.

\begin{tabular}{cccccccccc}
\hline Formulations & Disk 1 & Disk 2 & Disk 3 & Disk 4 & Disk 5 & Disk 6 & Mean & SD & P \\
\hline Test & 52.1 & 148.3 & 148.9 & 125.7 & 53.9 & 99.6 & 104.75 & 43.97 & \\
Conventional & 97.7 & 29.2 & 103 & 37.1 & 20.1 & 14.6 & 50.32 & 39.62 & \\
\hline
\end{tabular}

Table 3. Total micrograms of HT quantified for each skin disc in the two formulations evaluated: Test (liposome) vs. Reference in the Stratum Corneum and the Viable Epidermis.

\begin{tabular}{|c|c|c|c|c|c|c|c|c|c|c|c|c|}
\hline & \multicolumn{6}{|c|}{$\begin{array}{l}\text { Conventional } \\
\text { Formulation }(1 \% \text { cream })\end{array}$} & \multicolumn{6}{|c|}{$\begin{array}{l}\text { Test Formulation } \\
\text { (1\% Liposomecream) }\end{array}$} \\
\hline & DISK & DISK & DISK & DISK & DISK & DISK & DISK & DISK & DISK & DISK & DISK & DISK \\
\hline & 1 & 2 & 3 & 4 & 5 & 6 & 1 & 2 & 3 & 4 & 5 & 6 \\
\hline $\begin{array}{l}\text { Micrograms of } \\
\text { TH in the SC }\end{array}$ & 98.1 & 27.2 & 101.6 & 37.2 & 20.1 & 14.7 & 45.1 & 132.0 & 137.3 & 124.8 & 49.3 & 92.9 \\
\hline $\begin{array}{l}\text { Micrograms of } \\
\mathrm{TH} \text { in the VE }\end{array}$ & & 2.06 & 1.40 & & & & 6.88 & 16.49 & 11.73 & 1.08 & 4.47 & 6.82 \\
\hline
\end{tabular}

In Table 3, it can be seen that both formulations are present in a large part of the evaluated tapes, with the Liposome Test formulation being the one most frequently found in the SC and the viable epidermis. 
When comparing the penetration dynamics of both formulations (Conventional vs. Test), the differences between both formulations are clearly visible. The liposome test formulation not only managed to deliver the active ingredient to each of the layers of the SC studied, but also, when comparing the average in micrograms of TH present in the SC we can see that the amount of the drug with test formulation was greater than of the conventional formulation (Figure 3 ).

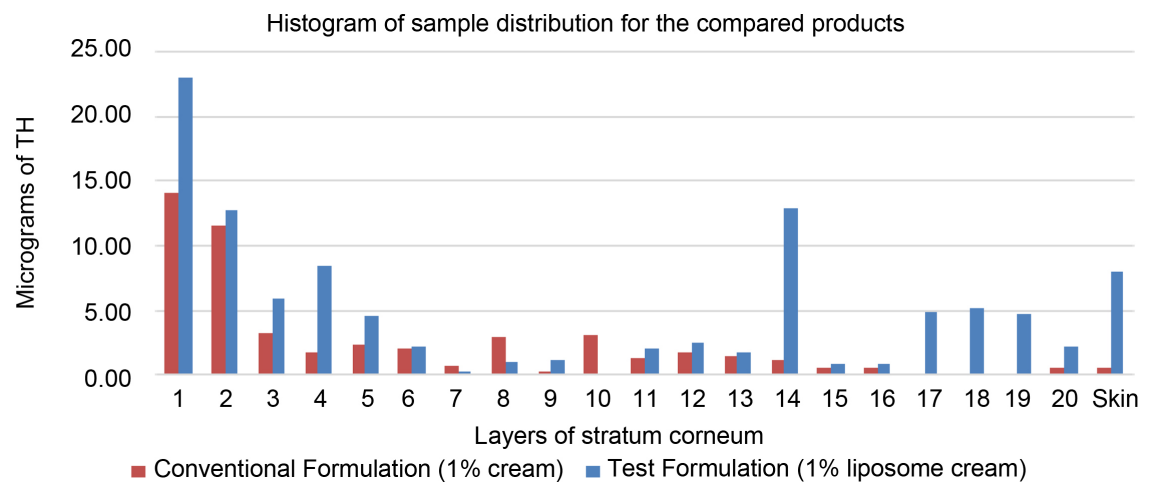

Figure 3. Comparison of the average micrograms of Terbinafine Hydrochloride, present in the Stratum Corneum, between the Conventional Formulation (Lamisil ${ }^{\circledR} 1 \%$ cream) vs. $1 \%$ liposome test formulation.

In both products tested (Test liposome vs. Conventional), comparisons were made based on the micrograms of TH present in the SC and the VE. The population mean, median value, standard deviation, maximum and minimum values, and the mass range for each of the formulations were estimated. The following table summarizes the values for each product.

\subsection{Comparative Statistic of the ex Vivo Test of Tape Stripping}

In the ex vivo tests, descriptive tests were carried out to confirm whether the differences found between the two formulations were statistically significant. Initially, logarithmic transformation of the data (LT) was performed in order to ensure the normality of the series. In both products evaluated, Reference Formulation vs. Test Formulation, comparisons were made based on the micrograms of reference TH present in the SC and the VE. For them, the average or microgram average for each product was estimated, the median value, the standard deviation, the maximum and minimum microgram values of the active ingredient in each product and the mass range for each one. The following table summarizes the values that describe each of the products (Table 4).

First, it was established that the two populations compared are independent, since they are samples that come from different pharmaceutical formulas that are not related to each other, second these variables are quantitative, independent and descriptive, so it was necessary to perform independent means comparison tests. The physicochemical characteristics of the $1 \%$ liposome Test formulation confer a considerable advantage over the Conventional formulation. 
Table 4. Descriptive statistics of the samples compared in the ex vivo assay based on the micrograms present in the SC and VE for the Conventional product (Lamisil ${ }^{\otimes} 1 \%$ cream) and test (TH liposome $1 \%$ cream).

\begin{tabular}{lcc}
\hline \multicolumn{1}{c}{ Variable } & Conventional TH 1\% Cream & TH liposome 1\% cream \\
\hline Mean $(\mu \mathrm{g}$ of TH) & 50.32 & 104.75 \\
Median & 33.15 & 112.65 \\
Standard deviation & 39.62 & 43.97 \\
Maximum value ( $\mu$ g of Terbinafine) & 14.00 & 23.02 \\
Minimum value ( $\mu$ g of Terbinafine) & 0.00 & 0.10 \\
Range ( $\mu$ g of Terbinafine) & 14.00 & 22.92 \\
\hline
\end{tabular}

\section{Discussion}

Penetration of the drug through the skin is usually limited by SC, it is a slow process, followed by rapid diffusion through the VE and the papillary dermis. Both processes are carried out by passive diffusion. The speed and magnitude of this transport are governed by Fick's law, according to which, the speed of diffusion is directly proportional to the diffusion coefficient and to the partition of the active principle and to its solubility in the aqueous medium surrounding the membrane, being inversely proportional to the thickness of the membrane to be traversed [21] [22] [23].

The TH has little solubility in water and is very lipophilic, which hinders its penetration into the SC. Because of this, pharmaceutical technology has designed new formulations based on nanometric structures such as liposomes, solid lipid nanoparticles, transferosomes, etosomes, etc., which act as SPF, which are also biodegradable and capable of encapsulating the active ingredients efficiently for the delivery of substances in terms of concentration and greater penetration in the different layers of the SC, because they increase the permeability and fluidity facilitating the transport of the active principle through membranes, they upgrade the bioavailability and control the delivery of drugs [24].

The in vitro tests with Franz Cells involve the diffusion processes of the substance to be evaluated through membranes from a donor compartment to a receptor compartment, which usually contains a buffer solution that simulates the extracellular fluid of animal tissues whose main objective is to guarantee the ideal physiological conditions. All the pharmacokinetic processes of absorption, distribution and elimination require the passage of the molecules of the drug through membranes (biological or synthetic). In vitro permeation studies are commonly used in trials that evaluate the delivery of drugs via topical [25] [26]. In those cases in which the solubility of the active principle is compromised, that is, it has very low solubility in inorganic solvents, as is the case of TH, substances such as ethanol, proteins and cyclodextrins deserve to be added to the diffusion medium to facilitate the exchange [14]. Fick's Laws on diffusion allow us to quantify through mathematical equations the process of diffusion of materials in a system that initially is not in equilibrium [21]. 
In this test, an active principle was designed that could cross the synthetic membrane and pass through a passive diffusion process from the donor compartment to the receptor compartment that contained the PBS buffer $\mathrm{pH} 5.8$ : Ethanol (1:2). When evaluating the curves of the obtained release profiles, it was possible to demonstrate that the TH managed to diffuse towards the moisturizing medium as a function of the gradient, observing differences between the Test and Reference formulation. With the Reference Formulation, a maximum of $20 \%$ diffusion was reached, $70 \%$ with the liposome Test Formulation. Trying to explain the mechanisms by which liposomes increase the permeation of HT through the synthetic membrane in the diffusion cells, we find that liposomes are used to promote the access of drugs to different cells. Liposomes are synthetic structures formed by one or more concentric bilayers of phospholipids that accommodate drugs inside water-soluble or liposoluble and macromolecules (such as enzymes, hormones, antigens, genetic material and other agents), whose composition facilitates access to cells with the capacity to trap them [27].

The dermis possesses a high water content and behaves as a barrier in drug penetration, mainly for those lipophilic components with a lipid/water partition coefficient greater than 600. It has been estimated that the SC provides a diffusional resistance of the penetration 1000 times higher for water-soluble drugs compared to that for fat-soluble drugs [28]. One of the most important parameters to consider in in vitro diffusion assays is the rate of diffusion of an analyte between the compartments. Additionally, they influence the recovery of the drug in the moisturizing medium, the lipophilic nature of the substance, that is, the more hydrophobic the substance, the less it diffuses. In addition, the higher its molecular weight or the volume of the substance could prevent passage through the pores of the membrane [28]. In this test the differences in the accumulated active concentrations $\left(\mu \mathrm{g} / \mathrm{cm}^{2}\right)$ observed between the Test and Reference formulation, taking into account the hydrophobicity of Terbinafine, indicate that in the Reference formulation there is greater resistance and lower affinity of the analyte with the medium, which generates a delay in its rate of diffusion, however in the liposome test formulation, the active principle is encapsulated within a lipid matrix that has polar heads (hydrophilic) disposed towards the outside by what favors passive diffusion. Drug transport systems based on lipid matrices such as liposomes increase the bioavailability of lipophilic drugs.

It has been found in the literature that solutions of saline phosphate buffer whose $\mathrm{pH}$ values are in ranges close to 5.8 modify the diffusion behavior of drugs. The non-ionized form will diffuse freely until it is balanced on both sides of the membrane, while the ionized form, due to its richness in hydrophilic groups, will not pass. When the membrane separates two media with different $\mathrm{pH}$ (e.g., blood relative to the intestinal lumen, urine, milk, saliva or prostatic fluid), an accumulation of the drug will occur on the side with the highest degree of ionization [29]. In this test the Reference Formulation (TH cream 1\%) was more exposed to the ionization by the medium, therefore, it generated that the 
active ingredient was retained in the matrix of the cream present in the donor compartment. While in the liposome test formulation, the vesicles generated a more stable internal environment that protected the terbinafine from ionization, favoring its diffusion to the receptor medium.

Among the critical parameters to evaluate the absorption of the active principle are the physicochemical characteristics of the drug that include solubility, how diffusion is carried out through the membranes described in the in vitro test, the characteristics of the pharmaceutical preparation such as particle size, product formulation, diffusion kinetics, etc. [23] [30] [31].

In this study, the ex vivo test of Tape Stripping for the evaluation of the penetration and quantification of the formulations, the tape strips contained the amount of corneocytes and the corresponding amount of the penetrated formulation, which was determined by a classical method of chromatography. The differences in the Test and Reference formulations strongly influenced the amount of SC removed with each strip of adhesive tape, as well as the amount of Terbinafine present in each layer of the SC.

In this test, it was evidenced that the Reference Formulation was mostly found in the first tapes extracted from the skin and in smaller quantity than the liposome Test Formulation expressed as $\mu \mathrm{g}$ of quantified active principle. On the other hand, the liposomated Test Formulation not only managed to reach a greater number of SC layers, but it also surpassed the Test Formulation in mass, reaching the $\mathrm{VE}$.

The SC is a permeability barrier with high lipophilic potential that hinders the penetration of drugs into the skin. The SC is represented as a network where the corneocytes, rich in keratin, are inside the intercellular matrix provided with lipids. From this organization, dermal permeation of compounds is given through the transcellular, intercellular and/or by cutaneous annexes. In this context, the use of DCS as topical liposomes is considered a potential option to increase the penetration and concentration of the drug through its release in the different layers of the skin, according to its formulation [6] [7]. It is described in the literature that topically applied drugs can cross the skin through different routes: intercellular, transcellular, transfolicular, via the sebaceous gland, via the sweat gland and mixed, being the intercellular route the most common. It has also been reported that non-polar drugs cross the SC through the intercellular route, while polar drugs cross the transcellular route [22] [28] [30]. Considering that the transcellular penetration depends to a great extent on the characteristics of the formulation, in this test we could observe that the physicochemical characteristics (Table 1) of the liposome Test Formulation (smaller particle size of the suspension of $72.25 \mathrm{~nm}$, the low polydispersity index (0.179), the acid $\mathrm{pH}(2.15)$ of the liposome emulsion) favored a better penetration in comparison with the larger molecules, as well as the condition of owning the active principle protected from ionization; because the nonionic molecules manage to penetrate better, they confirm that the characteristics of their formulation considerably 
favor the penetration of $\mathrm{TH}$ towards deeper layers of the non-viable epidermis and converting it into a much more efficient form in the delivery of the active principle. In this way, these DCS enhance the permeation of the active principle as it enters the SC by modifying the intercellular lipids, facilitating the penetration of hydrophilic drugs

On the other hand, according to the literature, the drug must be present at a concentration above its MIC in the action site or target for a guaranteed therapeutic effect [1] [3] [4] [5]. In this study, we observed that in each strip, except for strip 19 of the reference product, the quantities of Terbinafine are sufficient to obtain the MIC $(0.001-0.01 \mu \mathrm{g} / \mathrm{mL})$, observing that the average concentration of the Test formulation was higher than that of the Reference Formulation (Table 2), delimiting the MIC considered as a predictor of efficacy [1] [4] [5].

The existing differences regarding the physicochemical characteristics between both formulations were responsible for the differences found, being able to demonstrate that Terbinafina incorporated in SPF as liposomes, played an important role as an enhancing element of the skin penetration of this hydrophobic drug, because Its physicochemical characteristics facilitated its passage through the different routes of skin permeation.

In this study we could confirm that the in vitro and ex vivo assays were very suitable to obtain information on the homogeneity and distribution of both formulations in the SC and the VE, as well as highlighting the characteristics of the Liposomated Test formulation which confer it an important advantage over the Reference formulation (TH Cream 1\%).

\section{Conclusion}

Based on the above-captioned discussion, it can be concluded that the Test formulation (TH liposome 1\% cream) is superior to the Conventional formulation (Lamisil ${ }^{\circledR} 1 \%$ cream), in terms of diffusion and penetration at the level of the SC of the skin. The Terbinafine liposomes acted as a highly efficient Drug Carrier System that facilitates the delivery and permeability of the active principle through synthetic and natural membranes. This allows to reach Terbinafine values well above the reference MIC. Additionally, the DCS used was able to carry the drug Terbinafine through the skin, maintaining its size and load, optimizing its release in the target site of the skin (SC) where the pathogenic fungus is located.

\section{Conflicts of Interest}

The authors declare no conflicts of interest regarding the publication of this paper.

\section{References}

[1] Balfour, J.A. and Faulds, D. (1992) Terbinafine. A Review of Its Pharmacodynamic and Pharmacokinetic Properties, and Therapeutic Potential in Superficial Mycoses. 
Drugs, 43, 259-284. https://doi.org/10.2165/00003495-199243020-00010

[2] McClellan, K.J., Wiseman, L.R. and Markham, A. (1999) Terbinafine. An Update of Its Use in Superficial Mycoses. Drugs, 58, 179-202. https://doi.org/10.2165/00003495-199958010-00018

[3] Eman, A., et al. (2016) Skin Models for the Testing of Transdermal Drugs. Clinical Pharmacology, 8, 163-176. https://doi.org/10.2147/CPAA.S64788

[4] Year (2000) The Sanford Guide to Antimicrobial Therapy. 13th Edition, 75 p..

[5] Soares, M.M.S.R. and Cury, A.E. (2001) In Vitro Activity of Antifungal and Antiseptic Agents against Dermatophyte Isolates from Patients with Tinea Pedis. Brazilian Journal of Microbiology, 32, 130-134. https://doi.org/10.1590/S1517-83822001000200012

[6] Gaba, B., et al. (2015) Nanostructured Lipid Carrier System for Topical Delivery of Terbinafine Hydrochloride. Bulletin of Faculty of Pharmacy, Cairo University, 53, 147-159. https://doi.org/10.1016/j.bfopcu.2015.10.001

[7] Barry, B.W. (1983) Dermatological Formulations: Percutaneous Absorption. Journal Pharmaceutical Sciences, 73, 479. https://onlinelibrary.wiley.com/doi/abs/10.1002/jps.2600730442

[8] Rico, C.I., et al. (2013) Skin Permeation and Biodistribution of a Phthalocyanine Nanoemulsion of Chlorinated Aluminum (PcAlCl) Applied Topically in Wistar Rats. Argentine Journal of Dermatology, 94, 28.

https://rad-online.org.ar/2013/07/01/permeacion-en-piel-y-biodistribucion-de-unananoemulsion-de-ftalocianina-de-aluminio-clorada-pcalcl-aplicada-topicamente-en -ratas-wistar

[9] Nava, G., et al. (2011) Formulation and in Vitro, ex Vivo and in Vivo Evaluation of Elastic Liposomes for Transdermal Delivery of Ketorolac Tromethamine. Pharmaceutics, 3, 954-970. https://doi.org/10.3390/pharmaceutics3040954 https://www.ncbi.nlm.nih.gov/pmc/articles/PMC3857066/pdf/pharmaceutics-03-00 954.pdf

[10] Lampe, M.A., et al. (1983) Human Stratum Corneum Lipids: Characterization and Regional Variations. Journal of Lipid Research, 24, 120-130. http://www.jlr.org/content/24/2/120.full.pdf

[11] Jacobi, U., Taube, H., Schäfer, U.F., Sterry, W. and Lademann, J. (2005) Comparison of Four Different in Vitro Systems to Study the Reservoir Capacity of the Stratum Corneum. Journal of Controlled Release, 103, 61-71. https://doi.org/10.1016/j.jconrel.2004.11.013

[12] Escribano, E., Calpena, A.C., Queralt, J., Obach, R. and Doménech, J. (2003) Assessment of Diclofenac Permeation with Different Formulations: Anti-Inflammatory Study of a Selected Formula. European Journal of Pharmaceutical Sciences, 19, 203-210. https://doi.org/10.1016/S0928-0987(03)00103-9

[13] Mukherjee, B., et al. (2005) A Comparison between Povidone-ethylcellulose and Povidone-Eudragit Transdermal Dexamethasone Matrix Patches Based on in Vitro Skin Permeation. European Journal of Pharmaceutics and Biopharmaceutics, 59, 475-483. https://doi.org/10.1016/j.ejpb.2004.09.009

[14] Heike, W., Kostka, K.-H., Lehr, C.-M. and Schaefer, U.F. (2000) Drug Distribution in Human Skin Using Two Different in Vitro Test Systems: Comparison with in Vivo Data. Pharmaceutical Research, 17, 1475-1481. https://doi.org/10.1023/A:1007648807195

[15] Lademann, J., Jacobi, U., Surber, C., Weigmann, H.-J. and Fluhrc, J.W. (2009) The Tape Stripping Procedure-Evaluation of Some Critical Parameters. European Journal of Pharmaceutics and Biopharmaceutics, 72, 317-323. 
https://doi.org/10.1016/j.ejpb.2008.08.008

[16] Escobar-Chávez, J.J., et al. (2008) The Tape-Stripping Technique as a Method for Drug Quantification in Skin. Journal of Pharmacy \& Pharmaceutical Sciences, 11, 104-130. https://doi.org/10.18433/J3201Z

[17] Franz, T.J., Lehman, P.A. and Raney, S.G. (2009) Use of Excised Human Skin to Assess the Bioequivalence of Topical Products. Skin Pharmacology and Physiology, 22, 276-286. https://doi.org/10.1159/000235828

[18] Meyer, W., et al. (1978) The Skin of Domestic Mammals as a Model for the Human Skin, with Special Reference to the Domesticpig. Current Problems in Dermatology, 7, 39-52. https://doi.org/10.1159/000401274

[19] Schmook, F.P., et al. (2001) Comparison of Human Skin or Epidermis Models with Human and Animal Skin in in-Vitro Percutaneus Absorption. International Journal of Pharmaceutics, 215, 51-56. https://doi.org/10.1016/S0378-5173(00)00665-7

[20] Sekkat, N., et al. (2004) Porcineear Skin as a Model for the Assessment of Transdermal Drug Delivery to Prematureneonates. Pharmaceutical Research, 21, 1390-1397. https://doi.org/10.1023/B:PHAM.0000036912.94452.d0

[21] Debandi, M.V., et al. (2011) Evaluation of Different Membranes for in Vitro Release of Anticellulitic Actives. Advances in Science and Engineering, 2, 97-105.

[22] Villarino, N.F., et al. (2006) Transdermal Drug Administration: A Therapeutical Alternative. Analecta Veterinaria, 26, 28-37.

http://sedici.unlp.edu.ar/bitstream/handle/10915/11189/Documento_completo.pdf? $\underline{\text { sequence }=1}$

[23] Walters, K.A. (2002) Dermatological and Transdermical Formulations. Drugs and the Pharmaceutical Sciences. CRC Press, Boca Raton, Vol. 119, 89-115.

[24] Souto, E.B. and Muller, R.H. (2006) Investigation of the Factors Influencing the Incorporation of Clotrimazole in SLN and NLC Prepared by Hot High Pressure Homogenization. Journal of Microencapsulation, 23, 377-388. https://doi.org/10.1080/02652040500435295

[25] Chen, Y., et al. (2012) Development of Terbinafine Solid Lipid Nanoparticles as Tropical Delivery System. International Journal of Nanomedicine, 7, 4409-4418. https://doi.org/10.2147/IJN.S33682

[26] Nair, A., et al. (2013) Basic Considerations in the Dermatokinetics of Topical Formulations. Brazilian Journal of Pharmaceutical Sciences, 49, 423-434. https://doi.org/10.1590/S1984-82502013000300004

[27] Ozcan, I., et al. (2009) Enhanced Tropical Delivery of Terbinafine Hydrochloride with Chitosan Hydrogels. PharmSciTech, 10, 1024-1031.

https://doi.org/10.1208/s12249-009-9299-x

[28] Otarola, J.J., Lassalle, V., Fernández Band, B.S. and Garrido, M. (2012) Drug Carrier Systems Based on Nanostructured Lipid Carriers for Vehiculization of Diclofenac. http://www.mab4.uns.edu.ar/desc2.php?arc=014.pdf

[29] Volonté, M.G. and Quiroga, P. (2013) Pharmaceutical Analysis. Editorial of the University of La Plata. 1-27.

http://sedici.unlp.edu.ar/bitstream/handle/10915/32503/Documento_completo.pdf? sequence $=3$

[30] Hills, S., et al. (1992) An Investigation of the Pharmacokinetics of Topical Terbinafine. British Journal of Dermatology, 127, 322-326. https://doi.org/10.1111/j.1365-2133.1992.tb00461.x

[31] Walters, K.A. and Roberts, M.S. (2002) The Structure and Function of Skin. Marcel Dekker, New York, 1-40. 